Laurens R. Krol, Thorsten O. Zander

\title{
Towards a conceptual framework for cognitive probing
}

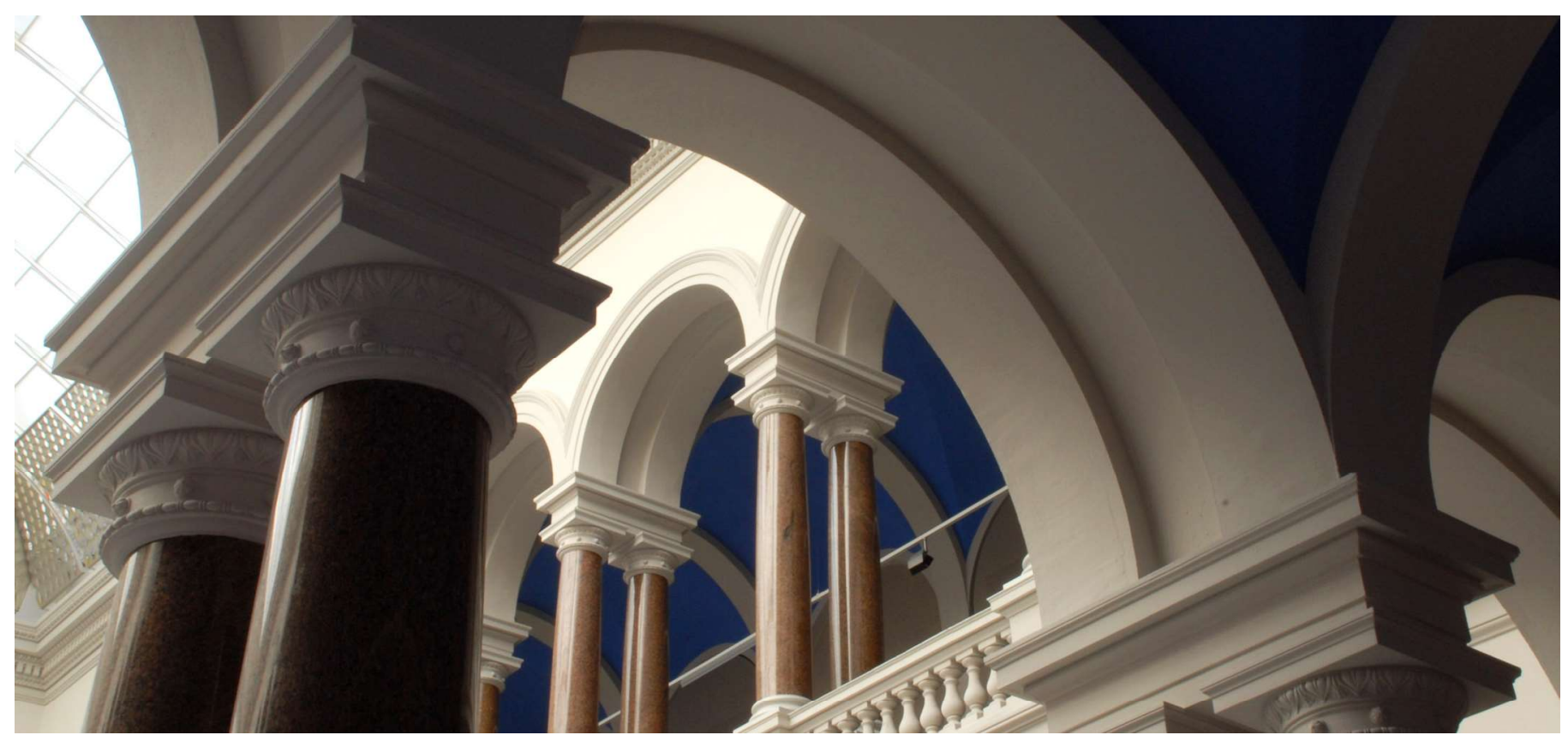

Krol, L. R. \& Zander, T. O. (2018). Towards a Conceptual Framework for Cognitive Probing. Symbiotic Interaction, 74-78. https://doi.org/10.1007/978-3-319-91593-7_8 


\title{
Towards a Conceptual Framework for Cognitive Probing
}

\author{
Laurens R. Krol ${ }^{1, \bowtie}$ and Thorsten O. Zander ${ }^{1}$ \\ ${ }^{1}$ Biological Psychology and Neuroergonomics, \\ Technische Universität Berlin, Berlin, Germany \\ lrkrol@gmail.com
}

\begin{abstract}
Cognitive probing combines the ability of computers to interpret ongoing measures of arbitrary brain activity, with the ability of those same computers to actively elicit cognitive responses from their users. Purposefully elicited responses can be interpreted in order to learn about the user, enable symbiotic and implicit interaction, and support neuroadaptive technology. We propose a working definition of cognitive probing that allows it to be generalised across different applications and disciplines.
\end{abstract}

Keywords: cognitive probing $\cdot$ brain-computer interface $\cdot$ neuroadaptive technology $\cdot$ implicit interaction $\cdot$ human-computer interaction

\section{Introduction}

A brain-computer interface (BCI) is a system that allows an output channel to be established directly between a user's brain and a technological system - an output channel "that is neither neuromuscular nor hormonal" [9]. This allows for example completely paralysed or locked-in patients to communicate with the outside world using mental spellers [1] or brain-activated prostheses [7]. Through BCI systems, people can control such devices using only their brain activity.

A passive brain-computer interface (pBCI) [14] uses similar hard- and software in order to interpret brain activity that was not meant to control a device. Instead, it detects and interprets "natural" [5] brain activity that reflects the user's cognitive and mental state, and uses this as implicit input to support ongoing human-computer interaction [12].

The automatic correction of user response errors is an early example of what is now known as pBCI. For example in a speeded reaction task, whenever an error negativity [3] was detected, the response would be undone [8]. This approach was later extended to machine errors: whenever the user observed the machine committing an error, it could be corrected if the appropriate brain signal was detected [13].

Note that these are indeed passive BCI applications, since the perception of such an error itself elicits the relevant brain activity, and the user expends no additional effort to inform the computer of the fact that an error occurred. 
This is the author's final preprint version of the following publication: Krol, L. R., \& Zander, T. O. (2018). Towards a conceptual framework for cognitive probing. In J. Ham, A. Spagnolli, B. Blankertz, L. Gamberini, \& G. Jacucci (Eds.), Symbiotic interaction (pp. 74-78). Cham: Springer International Publishing. doi: 10.1007/978-3-319-91593-7_8

The final publication is @ S Springer International Publishing AG, part of Springer Nature 2018

That machine errors elicit such a detectable response, confirmed also by other experiments [2], is a fact that can be actively exploited by the system. For example, the system can tentatively perform any number of random acts, and can then assess, using pBCI, whether or not these were perceived as erroneous or not by the user. Any action that was not perceived to be in error can then be definitively committed. As such, the user would have implicitly communicated to the system what they wanted it to do, without having given any explicit commands or instructions [11].

Such a scenario was recently demonstrated to be possible using a form of implicit cursor control $[11,15]$, and separately by another group using a robotic arm [4]. In this paper, we take the former as an example. Participants were observing the initially random movements of a cursor on a grid, on which one target location was indicated. For each movement, the computer could assess from ongoing measurements of brain activity whether or not that movement was perceived as either "acceptable" or "not acceptable". Using this information, the system learned over time which movements were apparently desired by the user, and adapted the cursor's behaviour in order to steer it towards the target location.

We believe that this approach, where the computer purposefully elicits responses in order to obtain information not explicitly communicated by the user, can be formulated more generally. The approach is not unique to the abovementioned example: armed with a general formulation we can see, in retrospect, that other, older applications have used this method as well. A standard definition of this approach should make it more easily recognisable as such, highlighting it as a worthwhile method of its own, and making it more accessible to other researchers across disciplines. We propose to name this approach cognitive probing.

\section{Cognitive Probing}

Cognitive probing refers to the general use of this approach. A stricter definition focuses on the defining behaviour of the system at hand: such a system utilises cognitive probes. We propose the following definition of a cognitive probe:

A cognitive probe is a single autogenous system adaptation that is initiated or co-opted by that system in order to learn from the user's contextual, cognitive brain response to it.

This definition consists of a number of terms that may warrant further discussion.

First of all, we use the term system adaptation to refer to any state change of the technological system $[5,6]$, be they the perceptible presentation of stimuli or feedback, or more subtle changes to the state or behaviour of the system. 
This is the author's final preprint version of the following publication: Krol, L. R., \& Zander, T. O. (2018). Towards a conceptual framework for cognitive probing. In J. Ham, A. Spagnolli, B. Blankertz, L. Gamberini, \& G. Jacucci (Eds.), Symbiotic interaction (pp. 74-78). Cham: Springer International Publishing. doi: 10.1007/978-3-319-91593-7_8

The final publication is @ Springer International Publishing AG, part of Springer Nature 2018

One such state change is autogenous when it is initiated by the system. This separates cognitive probes from adaptations whose specific form was decided by the human user, for example through explicit commands.

Such an autogenous state change can be either intended to be a probe - i.e. initiated primarily for that purpose - or, it can be a state change that occurs primarily for other reasons, for example, the presentation of feedback to inform the user. Such a latter adaptation may however still elicit a detectable brain response, can thus still be used for the same purpose - i.e., it can be co-opted to serve as a probe.

We are focusing in this definition on the user's cognitive brain response to these adaptations, as inferred from measures of their brain activity. The probes must thus in one way or another elicit cognition-related brain activity, or a change in ongoing brain activity.

Ultimately, the goal of cognitive probing is to obtain information from the user's brain response to the probes, either about the user, about a specific adaptation, or about the system as a whole. In short, the probes serve to learn.

For the gathered information to be meaningfully used as a basis for learning, further information is required. Not only the response itself must be known, but also, what elicited that response. That is the minimum possible context of the brain response. However, this context can be extended further to include other relevant contextual aspects: for example, the response may be dependent of the time of day, the physical location, or any other number of situational aspects.

\section{Discussion}

The example mentioned in the introduction fits the proposed definition of cognitive probing in the following way. Each single cursor movement was initiated by the computer itself, with the goal of eliciting a specific brain response. This response was then recorded in a user model that described the inferred user preferences in relation to the different possible movement directions. The system thus learned the user's preferred cursor behaviour. The observed improvement in the cursor's performance [15] demonstrates the effectiveness of this approach. In particular, the approach implemented here makes use of a sequence of probes. Where traditional BCI applications often use direct (open-loop or closed-loop [5]) adaptations based directly on single-trial brain responses, this example shows how multiple probes from a known context, combined with their (implicit) brain responses, can lead to inferences of higher aspects of cognition, in this case the desired cursor behaviour.

Note that the system could have learned this information even if the cursor continued to move randomly. However, the cursor used the obtained information in real time in order to reach its goal more quickly. This neuroadaptive behaviour of the cursor is not a necessity according to the proposed definition, although it illustrates how this approach can be used to increase the interactivity of humancomputer interaction, based entirely on implicitly communicated information 
This is the author's final preprint version of the following publication: Krol, L. R., \& Zander, T. O. (2018). Towards a conceptual framework for cognitive probing. In J. Ham, A. Spagnolli, B. Blankertz, L. Gamberini, \& G. Jacucci (Eds.), Symbiotic interaction (pp. 74-78). Cham: Springer International Publishing. doi: 10.1007/978-3-319-91593-7_8

The final publication is @ S Springer International Publishing AG, part of Springer Nature 2018

$[11,5]$. Such closed-loop interactions based on implicit input can provide the basis for a close, symbiotic relationship between humans and technology.

In the real world, we must deal not only with noisy environments, but also with a rich, uncontrollable context. Importantly, because the learning is based on implicit information and can be extended over longer periods of time, the single-trial accuracy of the system is not as critical as it would be for any sort of direct control application. Thus, this approach can be effective even with subperfect acquisition technology, such as more user-friendly dry electrodes [10]. Furthermore, even in varying environments and contexts, the primary context of interest is always known - it is the probe itself. Any contextual information that is added may be helpful, but is not required.

Because of this, we believe cognitive probing to be a promising strategy to be used for next-generation neuroadaptive technology, provided that it is used with due consideration and respect for the user's privacy of thought.

Acknowledgements Part of this work was supported by the Deutsche Forschungsgemeinschaft (ZA 821/3-1).

\section{References}

1. Birbaumer, N., Ghanayim, N., Hinterberger, T., Iversen, I., Kotchoubey, B., Kübler, A., Perelmouter, J., Taub, E., Flor, H.: A spelling device for the paralysed. Nature 398(6725), 297-298 (1999)

2. Chavarriaga, R., Sobolewski, A., Millán, J.d.R.: Errare machinale est: The use of error-related potentials in brain-machine interfaces. Frontiers in Neuroscience 8(208) (2014)

3. Falkenstein, M., Hoormann, J., Christ, S., Hohnsbein, J.: ERP components on reaction errors and their functional significance: a tutorial. Biological Psychology 51(2-3), 87-107 (2000)

4. Iturrate, I., Chavarriaga, R., Montesano, L., Minguez, J., Millán, J.d.R.: Teaching brain-machine interfaces as an alternative paradigm to neuroprosthetics control. Scientific Reports 5, 13893 (2015)

5. Krol, L.R., Andreessen, L.M., Zander, T.O.: Passive Brain-Computer Interfaces: A Perspective on Increased Interactivity. In: Nam, C.S., Nijholt, A., Lotte, F. (eds.) Brain-Computer Interfaces Handbook: Technological and Theoretical Advances, pp. 69-86. CRC Press, Boca Raton, FL, USA (2018)

6. Krol, L.R., Zander, T.O.: Passive BCI-based neuroadaptive systems. In: Proceedings of the 7th Graz Brain-Computer Interface Conference 2017. pp. 248-253 (2017)

7. Müller-Putz, G.R., Pfurtscheller, G.: Control of an electrical prosthesis with an SSVEP-based BCI. IEEE Transactions on Biomedical Engineering 55(1), 361-364 (2008)

8. Parra, L.C., Spence, C.D., Gerson, A.D., Sajda, P.: Response error correctiona demonstration of improved human-machine performance using real-time EEG monitoring. IEEE Transactions on Neural Systems and Rehabilitation Engineering 11(2), 173-177 (2003)

9. Wolpaw, J.R., Wolpaw, E.W.: Brain-computer interfaces: something new under the sun. In: Wolpaw, J.R., Wolpaw, E.W. (eds.) Brain-Computer Interfaces: Principles and practice, pp. 3-12. Oxford University Press, Oxford, UK (2012) 
This is the author's final preprint version of the following publication: Krol, L. R., \& Zander, T. O. (2018). Towards a conceptual framework for cognitive probing. In J. Ham, A. Spagnolli, B. Blankertz, L. Gamberini, \& G. Jacucci (Eds.), Symbiotic interaction (pp. 74-78). Cham: Springer International Publishing. doi: 10.1007/978-3-319-91593-7_8

The final publication is @ Springer International Publishing AG, part of Springer Nature 2018

10. Zander, T.O., Andreessen, L.M., Berg, A., Bleuel, M., Pawlitzki, J., Zawallich, L., Krol, L.R., Gramann, K.: Evaluation of a dry EEG system for application of passive brain-computer interfaces in autonomous driving. Frontiers in Human Neuroscience 11, 78 (2017)

11. Zander, T.O., Brönstrup, J., Lorenz, R., Krol, L.R.: Towards BCI-based Implicit Control in Human-Computer Interaction. In: Fairclough, S.H., Gilleade, K. (eds.) Advances in Physiological Computing, pp. 67-90. Springer, Berlin, Germany (2014)

12. Zander, T.O., Kothe, C.A.: Towards passive brain-computer interfaces: applying brain-computer interface technology to human-machine systems in general. Journal of Neural Engineering 8(2), 025005 (2011)

13. Zander, T.O., Kothe, C.A., Jatzev, S., Dashuber, R., Welke, S., De Filippis, M., Rötting, M.: Team PhyPA: Developing applications for brain-computer interaction. In: Proceedings of the Brain-Computer Interfaces for HCI and Games Workshop at the SIGCHI Conference on Human Factors in Computing Systems (CHI) (2008)

14. Zander, T.O., Kothe, C.A., Welke, S., Rötting, M.: Enhancing human-machine systems with secondary input from passive brain-computer interfaces. In: Proceedings of the 4th International Brain-Computer Interface Workshop \& Training Course. pp. 144-149. Verlag der Technischen Universität Graz, Graz, Austria (2008)

15. Zander, T.O., Krol, L.R., Birbaumer, N.P., Gramann, K.: Neuroadaptive technology enables implicit cursor control based on medial prefrontal cortex activity. Proceedings of the National Academy of Sciences 113(52), 14898-14903 (2016)

This conference contribution represents work in progress. See the following paper for updated work on cognitive probing: Krol, L. R., Haselager, P., \& Zander, T. O. (2020). Cognitive and affective probing: a tutorial and review of active learning for neuroadaptive technology. Journal of Neural Engineering, 17(1), 012001. doi: 10.1088/1741-2552/ab5bb5 